\title{
Experimental Effect Observation on Intensive Community STD Intervention
}

\author{
Song Shu-yi ${ }^{1, ~ *}$, Zhou Xiao-Ping ${ }^{1}$, Bai Wen-hai ${ }^{1}$, Qu Hui-ying ${ }^{1}$, Zhou Guo-mao ${ }^{2}$, Li Zhen ${ }^{2}$ \\ ${ }^{1}$ Shenzhen City Nanshan District People's Hospital, Huazhong University of Science and Technology Xiehe Shenzhen Hospital, Shenzhen, \\ China \\ ${ }^{2}$ Shenzhen City Nanshan District Chronic Disease Prevention and Control Institute, Shenzhen, China
}

Email address:

songsuccy@163.com (Song Shu-yi)

${ }^{*}$ Corresponding author

\section{To cite this article:}

Song Shu-yi, Zhou Xiao-Ping, Bai Wen-hai, Qu Hui-ying, Zhou Guo-mao, Li Zhen. Experimental Effect Observation on Intensive Community STD Intervention. International Journal of HIV/AIDS Prevention, Education and Behavioural Science. Vol. 4, No. 1, 2018, pp. $20-25$. doi: 10.11648/j.ijhpebs.20180401.14

Received: July 27, 2018; Accepted: August 14, 2018; Published: September 5, 2018

\begin{abstract}
Objective: To observe the implementation and effect of intensive community based sexually transmitted Disease (STD) intervention pilot in community. Methods: To adopt epidemiological experimental research method to establish the community experimental intervention group and the blank control group. To compare the intervention level and effect of venereal disease in each group after 1 years' work, and continue to follow up the pilot intervention group for next 2 years, and evaluated the pilot effect of intensive STD intervention. Results: During the first years of intervention, the two groups had no personnel changes, and there was no significant difference in the distribution of the two groups in the community population, the high-risk population and the vulnerable population $\left(\chi^{2}=1.864, \mathrm{P}=0.172\right)$. In the first year, 1141 cases of sexually transmitted diseases were screened, including 748 cases of clinical diagnosis and treatment, and 1306 cases of symptomatic treatment, all of which were significantly higher than those of the control group $\left(\chi^{2}=11.92,211.3,73.64 ; \mathrm{P}=0.001,0,0\right)$. In the intervention group of Neisseria gonorrhoeae and Candida, trichomonas, bacterial vaginosis positive screening rate significantly higher than that in control group $\left(\chi^{2}=4.45134 .85,48.7,17.08 ; \mathrm{P}=0.035,0,0,0\right)$. Intervention group of mycoplasma infection, condyloma acuminatum, Candida, trichomonas, bacterial vaginosis of 3, diagnosis and treatment effect significantly higher than that in control group $\left(\chi^{2}=17.6,4.055136 .8,48.7,17.08 ; \mathrm{P}=0,0.44,0,0,0\right)$. Two groups of pubic lice and scabies screening positive rate and the curative effect was $100 \%$, but the intervention group treatment 14 cases which were higher than the control group of 3 cases. The intervention group of urethral secretions and vaginal secretions of symptomatic management 3 days efficiency is higher than that of the control group $\left(\chi^{2}=86.377,37.239 ; \mathrm{P}=0,0\right)$. The number of STD clinics and the growth rate of total out-patient in intervention group were also significantly higher than those in control group $\left(\chi^{2}=82.87,7.44 ; \mathrm{P}=0,0.006\right)$. The $\mathrm{STD}$ screening, the average etiological treatment, and the average symptomatic management in the next two years was increased compared with the first year, and the differences were statistically significant $\left(\chi^{2}=49.619,12.559,107.437 ; \mathrm{P}=0,0,0\right)$ Conclusion: The effect of intensive STD intervention is much better than the current STD intervention service. Intensive STD intervention has good sustainability and is suitable to be popularized in urban community health service.
\end{abstract}

Keywords: Intensive, Community, Sexually Transmitted Disease (STD), Pilot, Effect

\section{Introduction}

Taking advantage of community health service to carry out Sexually transmitted Disease (STD) intervention is an expert consensus to move forward AIDS/STD intervention [1-3]. Over the past decade, our city has also actively participated in some major international and regional STD intervention projects, such as Sino British, Sino European and Sino Australian projects [1-3]. The practical experiences show that the effect and sustainability of the community STD intervention are influenced by many practical conditions, mainly related to the factors such as community needs, 
personnel, outpatient volume, work flow, collaboration support and so on [4-6]. Therefore, we design and adopt intensive STD intervention to observe the effect of community pilot, and compare the results of epidemiological experiments. The results are reported as follows.

\section{Subjects and Methods}

\subsection{Subjects}

The target population of the STD intervention work. 1) High-risk: Patients with symptoms of STDs, sexual services and their customers; 2) Vulnerable: High-risk sexual behavior within 3 months, migrant workers, and migrant temporary workers in service industries.

\subsection{Methods}

\subsubsection{Group Observation}

Choose two communities with higher STD epidemics reported in 10 years in the district as pilot sites [7-8]. The two communities were divided into two groups, the pilot experimental intervention group and the blank control group. To compare the intervention effect in the two groups of their own community health service centers (CHSC). The two groups were observed for 1 year, and the intervention group was followed up for next 2 years.

\subsubsection{Measures for the Intervention of STD in Each Group}

(1) Pilot experimental intervention group: Work under the guidance of professional institutions or the guidelines [9]. The department of AIDS prevention of the CDC, the department of STDs of institute of chronic diseases prevention and treatment the STD laboratory of the hospital headquarters and other related departments are responsible for quality control and standard correction. 1) Outreach activities: The assistant doctor is responsible for organizing doctors, nurses, and social workers to form outreach teams of 2 to 5 people each time. Job content: a. Community STD knowledge publicity, personal consultation, guidance and behavior intervention, mobilization screening; $b$. Blood collection and inspection on the spot. During the first year, STD outreach services were conducted regularly 1 times every 2 weeks, and in the next two year were carried out on demand. 2) Screening test [10], Follow up management: a. Screening: Conducting the initial screening in the community health center, or sent to the corresponding sexually transmitted diseases laboratory. The nurse is responsible for blood collection and result notification. The physician assistant is responsible for sexual partner tracking and follow-up visits. b. Detective methods: Get and submit blood samples of human immunodeficiency virus (HIV) and syphilis. Neisseria gonorrhoeae infection was detected by microscopic examination of smear Gram stain and polymerase chain reaction (PCR). For female suspected gonococcal infection, Neisseria gonorrhoeae culture was applied. Chlamydia trachomatis infection in the genital tract using latex antigen detection; Mycoplasma infection (ureaplasma urealyticum infection) using culture method; Bacterial vaginosis using dry chemical enzymatic method; Trichomonas vaginitis using hanging drop method; Candida infection using wet Tablet test; And carry out haze and acne examination; Condyloma using white acetic acid test for clinical examination. c. Follow-up monitoring: According to the standard, set up observation time for different STDs and carry out case management. 3) Clinical Services [11-13]: To be treated by a doctor and assisted by a nurse. a. Diagnosis: etiological diagnosis, clinical diagnosis and experience diagnosis. b. Treatment [12]: etiological treatment, symptomatic management/ clinical experience treatment [13], or refer to a specialist or a centralized doctor.

(2) Blank control group: Continue to use the current STD service method, that is: 1) Outpatient screening: subjects with a history of suspected sexually transmitted infections; fertility services and high-risk individuals found during free medical examinations for persons over the age of 65 . Outpatient etiology detection and inspection items and methods are the same as the pre-set group. 2) Clinical intervention: According to reference guidelines [11] for STD diagnosis and clinical intervention. HIV, syphilis voluntary screening, diagnosis and treatment of sexually transmitted diseases are difficult to refer to the hospital headquarters.

\subsection{Statistical Analysis}

STD service data are derived from the outpatient service data of Shenzhen community health service electronic information system, and all statistical data were checked and supplemented by the Community health service management center. SPSS18.0 software was used for statistical analysis.

\section{Results}

\subsection{Two Groups of Comparison}

\subsubsection{Baseline Survey [14] of STD Services}

The intervention group had 59,400 of the community population, of which about 3,164 of the target population, of whom 329 were high-risk people and 2,835 were vulnerable people. The control group had 49,800 of the community population, of which about 3,801 of the target population, of whom 358 were high-risk people and 3,443 were vulnerable pelple. There is no statistical difference between the two groups of target populations $\left(\chi^{2}=1.864, \mathrm{P}=0.172\right)$. The population composition of the two groups in the community is shown in Table 1. 
Table 1. The population composition of the two groups.

\begin{tabular}{|c|c|c|c|c|c|c|}
\hline \multirow{2}{*}{ Characteristics } & \multicolumn{2}{|c|}{ The intervention group } & \multicolumn{2}{|c|}{ The control group } & \multirow{2}{*}{$\chi^{2}$} & \multirow{2}{*}{$\mathbf{P}$} \\
\hline & Case number & Percentage $/ \%$ & Case number & Percentage $/ \%$ & & \\
\hline Population & 59400 & & 49800 & & & \\
\hline Male & 29100 & 49.00 & 24290 & 48.78 & 0.456 & 0.500 \\
\hline Female & 30300 & 51.00 & 25500 & 51.20 & & \\
\hline \multicolumn{7}{|c|}{ Household registration } \\
\hline Local & 2800 & 4.71 & 2270 & 4.55 & 1.799 & 0.407 \\
\hline Resident & 40800 & 68.69 & 34300 & 68.88 & & \\
\hline \multicolumn{7}{|l|}{ Age } \\
\hline$\leq 14$ & 11390 & 19.18 & 10130 & 20.34 & 26.967 & 0.000 \\
\hline $15 \sim 59$ & 46380 & 78.08 & 38420 & 77.15 & & \\
\hline$\geq 60$ & 1625 & 2.74 & 1250 & 2.51 & & \\
\hline
\end{tabular}

Notes: The resident population includes local population.

\subsubsection{STDs Screening}

The intervention group conducted a total of 1141 STD pathogenic screening, 561 positive cases were detected. The control group conducted a total of 466 screening, 185 positive cased were detected. The difference was statistically significant $\left(\chi^{2}=11.92, \mathrm{P}=0.001\right)$, See Table 2 for details.
Combined with the baseline population of the two groups, relative to the intervention group, about $72 \%$ of the STD cases in the control group were missed screening. In the intervention group, 47 cases were screened by referral, of them 34 cases were lost to visit. The loss rate was $72.34 \%$.

Table 2. Statistical table of etiological screening

\begin{tabular}{|c|c|c|c|c|c|c|c|c|}
\hline \multirow{2}{*}{$\begin{array}{l}\text { Etiological detection } \\
\text { of STD }\end{array}$} & \multicolumn{3}{|c|}{ The intervention group } & \multicolumn{3}{|c|}{ The control group } & \multirow{2}{*}{$\chi^{2}$} & \multirow{2}{*}{$\mathbf{P}$} \\
\hline & test number & positive number & Positive rate\% & test number & positive number & Positive rate\% & & \\
\hline $\mathrm{HIV}-\mathrm{Ab}$ & 87 & 0 & 0.00 & 2 & 0 & 0 & 1 & 1 \\
\hline Syphilis & 82 & 5 & 6.09 & 2 & 0 & 0 & 0.249 & 0.618 \\
\hline Gonococcus culture & 13 & 9 & 69.23 & 19 & 7 & 36.84 & 3.239 & 0.072 \\
\hline Chlamydia & 78 & 16 & 20.51 & 17 & 2 & 11.76 & 0.696 & 0.404 \\
\hline Mycoplasma & 78 & 41 & 52.56 & 34 & 16 & 47.06 & 0.287 & 0.592 \\
\hline Gonococcus & 41 & 27 & 65.85 & 0 & 0 & 0.00 & 4.450 & 0.035 \\
\hline Trichomonas vaginitis & 107 & 99 & 92.52 & 21 & 6 & 28.57 & 48.7 & 0.00 \\
\hline Bacterial vaginosis & 219 & 157 & 71.69 & 64 & 28 & 43.75 & 17.08 & 0.00 \\
\hline Pubic louse + scabies & 14 & 14 & 100.00 & 3 & 3 & 100.00 & 1 & 1 \\
\hline
\end{tabular}

\subsubsection{STD Clinical Service}

(i) Etiological diagnosis and treatment [12, 15] According to the requirements of the Shenzhen Health Standard, without improvement of 3 days treatment, the community health center should referral the patient to hospital. So we took the observation that the symptoms were relieved or disappeared in three days as the effective. In the intervention group, A total of 748 people were took etiological treatment, and of whom 649 were effective; In the control group, 353 were took etiological treated, and of whom 160 were effective. The difference between the two groups was statistically significant $\left(\chi^{2}=211.3\right.$; $\mathrm{P}=0.000)$, See Table 3 for details. In the intervention group, 113 patients were referred to STD specialists for treatment and 12 were lost. The rate of lost interviews was $10.62 \%$. In the intervention group, the effective rate of gonorrhea treatment was not high, which was related to many complications and complicated condition of the patients [16]. It also showed that the diagnosis and treatment of gonorrhea was relatively difficult.

Table 3. Statistical comparison of etiological diagnosis and treatment.

\begin{tabular}{|c|c|c|c|c|}
\hline \multirow{2}{*}{ Classification of infection } & \multicolumn{2}{|l|}{ Effective rate } & \multirow{2}{*}{$\chi^{2}$} & \multirow{2}{*}{$\mathbf{P}$} \\
\hline & The intervention group & The control group & & \\
\hline Gonorrhea & $72.50(29 / 40)$ & $100.00(2 / 2)$ & 0.745 & 0.388 \\
\hline Chlamydia infection & $82.05(64 / 78)$ & $70.59(12 / 17)$ & 1.146 & 0.284 \\
\hline Mycoplasma infection & $91.03(71 / 78)$ & $59.09(26 / 44)$ & 17.610 & 0.000 \\
\hline Condyloma & $82.93(34 / 41)$ & $0(0 / 2)$ & 4.055 & 0.044 \\
\hline Genital herpes & $76.47(13 / 17)$ & $33.33(1 / 3)$ & 1.556 & 0.212 \\
\hline Candidiasis & $89.94(438 / 487)$ & $51.09(117 / 229)$ & 136.880 & 0.000 \\
\hline Bacterial vaginosis & $71.69(157 / 219)$ & $43.75(28 / 64)$ & 17.080 & 0.000 \\
\hline Pubic louse + scabies & $100.00(14 / 14)$ & $100.00(3 / 3)$ & 1 & l \\
\hline
\end{tabular}


(ii) Non pathogenic diagnosis and treatment [13, 17, 18] For suspected patients without screening, according to clinical symptoms, were treated by symptomatic Management or clinical experience. In the intervention group, 1306 cases were treated and 1018 cases were effective; In the control group, 882 cases were treated and 538 cases were effective. The difference between the two groups was statistically significant. $\left(\chi^{2}=73.64 ; \mathrm{P}=0.000\right)$, See Table 4 for details.

Table 4. Statistical table of the effective rate of symptomatic treatment cases.

\begin{tabular}{|c|c|c|c|c|c|c|c|c|}
\hline \multirow{2}{*}{$\begin{array}{l}\text { Symptomatic management/clinical experience } \\
\text { treatment }\end{array}$} & \multicolumn{3}{|c|}{ The intervention group } & \multicolumn{3}{|c|}{ The control group } & \multirow[b]{2}{*}{$\chi^{2}$} & \multirow[b]{2}{*}{$\mathbf{P}$} \\
\hline & Total cases & $\begin{array}{l}\text { Effective } \\
\text { number }\end{array}$ & $\begin{array}{l}\text { Effective } \\
\text { rate } / \%\end{array}$ & Total cases & $\begin{array}{l}\text { Effective } \\
\text { number }\end{array}$ & $\begin{array}{l}\text { Effective } \\
\text { rate } / \%\end{array}$ & & \\
\hline Abnormal vaginal discharge/vaginitis/cervicitis & 654 & 497 & 75.99 & 364 & 206 & 56.59 & 37.239 & 0.000 \\
\hline Genital ulcers/herpes/warts & 62 & 15 & 24.19 & 38 & 7 & 18.42 & 0.457 & 0.499 \\
\hline Scrotal swelling/epididymal inflammation, etc & 13 & 3 & 23.08 & 14 & 1 & 7.14 & 1.356 & 0.244 \\
\hline Buboes/buboes lymphadenitis & 2 & 2 & 100.00 & 3 & 0 & 0 & 5 & 0.025 \\
\hline
\end{tabular}

\subsubsection{The Outpatient Volume in Two Groups}

During the first 1 year of the intervention group, the number of STD visits in the community health center increased from 989 to 2,430 , while the control group increased from 527 to 694. It shows Since the pilot work, STD services have increased in both groups, the intervention group is higher than the control group, the difference is statistically significant $\left(\chi^{2}=82.87, \mathrm{P}=0.000\right)$. The number of outpatients in the intervention group increased from 20,321 to 22,188 , and the control group increased from 17,019 to 17,864 . The growth rate of outpatients in the intervention group was higher than that in the control group. The difference was statistically significant $\left(\chi^{2}=7.44, \mathrm{P}=0.006\right)$.

\subsection{Self Comparison in Intervention Group}

\subsubsection{STD Screening and Education}

In the first year of the intervention group, there were 24 STD outreach activities, 2,100 consultation sessions, 1,300 sexual partners notification and follow-up. In the next two years, there were 9 STD outreach activities, more than 1400 consulted persons, more than 2,900 times of sexual partners' notification and follow-up. In the first year of intervention, 1,141 were screened, 561 were positive, 2,161.5 cases per year were screened in the next two years, and 758 cases per year were positive. The STD screening in the next two years was increased compared with the first year, and the difference was statistically significant. $\left(\chi^{2}=49.619 ; \mathrm{P}=0.000\right)$, See Table 5 for details.

Table 5. Statistical table of etiological screening in intervention group.

\begin{tabular}{|c|c|c|c|c|c|c|c|c|}
\hline \multirow{2}{*}{ Classification of infection } & \multicolumn{3}{|l|}{ First year } & \multicolumn{3}{|c|}{ Annual average of second, third years } & \multirow{2}{*}{$\chi^{2}$} & \multirow{2}{*}{$\mathbf{P}$} \\
\hline & Test number & Positive number & $\%$ & Test number & Positive number & $\%$ & & \\
\hline $\mathrm{HIV}-\mathrm{Ab}$ & 87 & 0 & 0.00 & 96.5 & 1 & 1.04 & 1 & $1.000^{\mathrm{a}}$ \\
\hline Syphilis & 82 & 5 & 6.09 & 96.5 & 2.5 & 2.59 & $1.143^{b}$ & 0.285 \\
\hline Gonococcus culture & 54 & 9 & 69.23 & 52 & 30.5 & 58.65 & 25.394 & 0.000 \\
\hline Chlamydia & 78 & 16 & 20.51 & 245.5 & 23.5 & 9.57 & 21.718 & 0.000 \\
\hline Mycoplasma & 78 & 41 & 1.00 & 255.5 & 100.5 & 39.33 & 4.893 & 0.035 \\
\hline Monilial infection & 107 & 99 & 92.52 & 225.5 & 162.5 & 71.84 & 19.842 & 0.000 \\
\hline Trichomonas vaginitis & 219 & 157 & 71.69 & 548.5 & 154.5 & 28.17 & 151.187 & 0.000 \\
\hline Bacterial vaginosis & 14 & 14 & 100 & 13.5 & 13.5 & 100 & l & l \\
\hline
\end{tabular}

Note: $\mathrm{a}$ is the $\mathrm{P}$ value of the Fisher exact probability method, $\mathrm{b}$ is the $\chi 2$ of the correction method.

\subsubsection{STD Clinical Service}

In the first year of the intervention group, 748 patients were took the etiological treatment, and 649 were effective. In the following two years, 963 patients per year were treated, and 870.5 patients per year were effective. The average treated cases per year in the following two years was more than that in the first year, and the difference was statistically significant $\left(\chi^{2}=12.559, \mathrm{P}=0.000\right)$, See Table 6 for details. In the first year, 1,306 patients were treated with Symptomatic management and 1018 were effective. The following two years were 1,679 and 1,253 were effective. The total number of cases in the following two years was more than that in the first year. The difference was statistically significant $\left(\chi^{2}=107.437, \mathrm{P}=0.000\right)$, see Table 7 for details. 
Table 6. Statistical table of etiological diagnosis and treatment in intervention group.

\begin{tabular}{|c|c|c|c|c|c|c|c|c|}
\hline \multirow{2}{*}{$\begin{array}{l}\text { Classification of } \\
\text { infection }\end{array}$} & \multicolumn{3}{|l|}{ First year } & \multicolumn{3}{|c|}{ Annual average of second, third years } & \multirow{2}{*}{$\chi^{2}$} & \multirow{2}{*}{$\mathbf{P}$} \\
\hline & Total cases & effective cases & $\%$ & Total cases & effective cases & $\%$ & & \\
\hline Gonorrhea & 40 & 29 & 72.5 & 36 & 30 & 83.33 & 1.849 & 0.223 \\
\hline Chlamydia infection & 78 & 64 & 82.05 & 28 & 23 & 82.14 & 0.000 & 1.000 \\
\hline Mycoplasma infection & 78 & 71 & 91.02 & 291 & 216 & 74.23 & 10.707 & 0.001 \\
\hline Condyloma & 41 & 34 & 64.28 & 37 & 30 & 81.08 & 0.060 & 1.000 \\
\hline Genital herpes & 17 & 13 & 70.58 & 41 & 28 & 68.29 & 0.446 & 0.576 \\
\hline Candidiasis & 487 & 438 & 90.14 & 1005 & 895 & 89.05 & 0.318 & 0.625 \\
\hline
\end{tabular}

Table 7. Statistical table for medical treatment of intervention group.

\begin{tabular}{|c|c|c|c|c|c|c|c|c|}
\hline \multirow{2}{*}{$\begin{array}{l}\text { Symptomatic management/clinical experience } \\
\text { treatment }\end{array}$} & \multicolumn{3}{|c|}{ The intervention group } & \multicolumn{3}{|c|}{ The control group } & \multirow[b]{2}{*}{$\chi^{2}$} & \multirow[b]{2}{*}{$\mathbf{P}$} \\
\hline & Total cases & $\begin{array}{l}\text { Effective } \\
\text { number }\end{array}$ & $\begin{array}{l}\text { Effective } \\
\text { rate } / \%\end{array}$ & Total cases & $\begin{array}{l}\text { Effective } \\
\text { number }\end{array}$ & $\begin{array}{l}\text { Effective } \\
\text { rate } / \%\end{array}$ & & \\
\hline Abnormal urinary secretion and dysuria/urethritis & 393 & 378 & 96.18 & 330 & 77 & 66.38 & 522.996 & 0.000 \\
\hline Abnormal vaginal discharge/vaginitis/cervicitis & 654 & 497 & 75.99 & 774.5 & 589 & 76.05 & 0.001 & 1.000 \\
\hline Genital ulcers/herpes/warts & 62 & 15 & 24.19 & 56 & 23 & 41.07 & 4.993 & 0.031 \\
\hline Female lower abdomen pain/pelvic inflammation & 182 & 123 & 67.58 & 242.5 & 177 & 72.99 & 1.899 & 0.178 \\
\hline Scrotal swelling/epididymal inflammation, etc & 13 & 3 & 23.07 & 9.5 & 3 & 31.58 & $0.016^{\mathrm{a}}$ & 0.900 \\
\hline
\end{tabular}

Note: $\mathrm{a}$ is the $\chi^{2}$ of the correction method.

\section{Conclusion}

The pilot intervention group and the blank control group were similar in terms of community conditions, management mechanism and number of employees. The outpatient volume and performance levels of the two groups before the trial were higher than those of the district average. After intensive STD intervention, the efficacy of the intervention group was significantly better than that of the control group. The reasons are as follows: 1) The outreach activities based on baseline surveys, together with professional STD laboratory support and peer education services [19], met the needs of STD high-risk population for primary care and professional services, effectively promoted STD screening, consultation and other services, and were superior to voluntary counseling and testing point [10]. 2). The intervention group expanded STD symptomatic treatment program for asymptomatic high-risk groups, effectively managed the false negative and refused screening of pathogens $[17,18]$, giving full play to the application value of symptomatic treatment at the grass-roots level. 3) Local Screening or blood sampling is conducive to community STD management. For neisseria gonorrhoeae screening needs referral, there were more visits missed and fewer screening, and the intervention is insufficient. The project believes that, unlike previous experience in China [4-6], intensive STD intervention is implemented by general practitioners and their teams, which has a workflow adapted to CHSCs [20], can be carried out in CHSCs with a large per capita outpatient volume, and can maintain the momentum for sustained STD intervention. After one year of pilot observation, the outpatient volume and performance level of the intervention group were still higher than that of the control group. Of the intervention group, the annual average STD intervention service volume and service level in the following two years was even higher than that in the previous year. The analysis is, through the existing CHSC outpatient service compensation mechanism, even in the absence of special funds to support the situation, the continuous development of community STD intervention is supported by the performance improvement resulting from the community demand and outpatient volume growth brought about by intensive STD intervention services. It shows that intensive STD intervention has a good sustained operability and is suitable for popularization in urban community health services.

\section{Acknowledgements}

The authors want to extend their thanks to The Science and Technology Innovation Fund of Shenzhen City (JCYJ20140411093600193) for it's support.

\section{References}

[1] Wang Quanpei, Ye Ganyun, Gong Xiangdong, etc. Future thinking on prevention and treatment of STDs in China [J]. Chinese AIDS STD, 2004, 10(3): 223-224, 233.

[2] Hao Yang, Shan Duo, Fu Xiaojing, etc. Feasibility analysis of community health service centers participating in AIDS prevention and treatment $[\mathrm{J}]$. Chinese Journal of Preventive Medicine, 2014 (05):386-390.

[3] Low N, Broutet N, Adu-Sarkodie Y, et al. Global control of sexually transmitted infections [J]. Lancet, 2006:368-2001.

[4] Meng Sha, Liu Min. Integrating AIDS/STD prevention services into urban community health service case studies $[\mathrm{J}]$. Chinese General Practice Medicine, 2009, 12(6A): 1037-1038.

[5] Li Yanqi. Survey of AIDS Resources in Shanghai Community Health Service Center [J]. Preventive medicine in Southern China, 2010(6):74-76.

[6] Gong Tao. Report on the intervention of migrants' behavior in the streets of Kangjian in 2014 [J]. Chinese community physicians, 2014, 30(30):171-172. 
[7] Li Lan, Li Zhen, Yuan Jun, etc. Nanchang District, Shenzhen, 2009 STD analysis of the epidemic [J]. Occupation and Health, 2011, 27(18): 2112-2114.

[8] Hong Xuan, Sun Fengxi, Liu Fengying, etc. Epidemiological analysis of venereal disease in Guangdong Province, 2004 2012 [J]. Chinese Journal of Department of Dermatology, 2014, 21(1): 65-68.

[9] Management measures for STD prevention and control [M]. Ministry of Health, PRC, 2012.

[10] Gong Xiangdong, Yue Xiaoli, Teng Fei, etc. Baseline investigation and analysis of laboratory detection status of STDs in China [J]. Chinese AIDS STD, 2010, 16(2):99-104.

[11] Standardized service and management standards for STD clinic [C]. Venereal control center of CDC. 2010.

[12] Wang Qianqiu, Liu Dengzhong, Xu Lihua. STDs clinical diagnosis and treatment intervention guide [M]. Shanghai: Shanghai Science and Technology Press, 2014:91-162.

[13] E Boonstra, M Lindbk. Symptomatic management of sexually transmitted diseases in Botswana's primary health care: quality of care aspects $[\mathrm{J}]$. Tropical Medicine and International Health, 2003, 8(7):604-614.

[14] Lin Zhihong, Song Shuyi, Bai Wenhai. Investigation on the high risk population of venereal disease in Nan yuan community [J]. Nursing Research, 2017, 31(31):4024-4025.
[15] Shang Shuxian, Wu Xiaochu. Guidelines for diagnosis and treatment of syphilis, gonorrhea, genital herpes, Chlamydia trachomatis infection in genital tract $[\mathrm{J}]$. Chinese Journal of Dermatology, 2014, 47(5):365-372.

[16] Hack JB, Hecht C. Emergency physicians' patterns of treatment for presumed gonorrhea and chlamydia in women: one center' s practice. Emerg Med, 2009, 37(3): 257-263.

[17] Liu Min, Zeng Guang, Zhang Linghua, etc. Epidemiological investigation of female genital tract infection and exploratory study on treatment of vaginal secretions [J]. Chinese Journal of Epidemiology, 2002, 23(6):422-426.

[18] Zhao Jin, Li Wei, Li Dexian, etc. Analysis of factors influencing the popularization and application of venereal disease treatment technology [J]. Guangxi Medical, 2011, 33(3): 365-366.

[19] Xi Yan, Tang Haiqin. Status quo of training and diagnosis and treatment of STD related diseases among primary medical staff [J]. China's Health Industry, 2017, 25(11):11-14.

[20] Liang Yuanfei, Chen Yuanqing, Yao Gongyuan, etc. Analysis of the effect of behavioral intervention on high-risk groups in community entertainment places combined with standardized venereal clinic $[\mathrm{J}]$. South of the Five Ridges Journal of the Department of Dermatology and Venereal Diseases. 2007 14(1):56-58. 